\title{
Early Experience of Ramucirumab Therapy in Japanese Patients With Unresectable Hepatocellular Carcinoma in Real-World Practice
}

Yuka Hayakawa ( $\nabla$ y.hayakawa@musashino.jrc.or.jp )

Musashino Red Cross Hospital

Kaoru Tsuchiya

Musashino Red Cross Hospital

Masayuki Kurosaki

Musashino Red Cross Hospital

Yutaka Yasui

Musashino Red Cross Hospital

Shun Kaneko

Musashino Red Cross Hospital

Kento Inada

Musashino Red Cross Hospital

Sakura Kirino

Musashino Red Cross Hospital

Koji Yamashita

Musashino Red Cross Hospital

Leona Osawa

Musashino Red Cross Hospital

Shuhei Sekiguchi

Musashino Red Cross Hospital

Mayu Higuchi

Musashino Red Cross Hospital

Kenta Takaura

Musashino Red Cross Hospital

Chiaki Maeyashiki

Musashino Red Cross Hospital

Nobuharu Tamaki

Musashino Red Cross Hospital

Hiroyuki Nakanishi

Musashino Red Cross Hospital

Jun Itakura 
Musashino Red Cross Hospital

\section{Yuka Takahashi}

Musashino Red Cross Hospital

\section{Daiei Takahashi}

Musashino Red Cross Hospital

\section{Yasuhiro Asahina}

Tokyo Medical and Dental University

\section{Ryuichi Okamoto}

Tokyo Medical and Dental University

\section{Namiki Izumi}

Musashino Red Cross Hospital

\section{Research article}

Keywords: hepatocellular carcinoma, ramucirumab, alpha fetoprotein, adverse events

Posted Date: November 20th, 2020

DOl: https://doi.org/10.21203/rs.3.rs-111378/v1

License: (c) (1) This work is licensed under a Creative Commons Attribution 4.0 International License. Read Full License 


\section{Abstract}

\section{Background}

We aimed to investigate the efficacy and safety of ramucirumab therapy in Japanese patients with unresectable hepatocellular carcinoma (u-HCC) in real-world practice.

\section{Methods}

A total of 16 patients with u-HCC were treated with ramucirumab of $\geq 2$ cycles between July 2019 and April 2020. Modified response evaluation criteria in solid tumors (RECIST) and RECIST version 1.1 were used to evaluate radiological responses.

Results

The patients received ramucirumab as second-line $(n=4)$, third-line $(n=2)$, and fourth-line $(n=10)$. Median observation period was 3.7 months and 3 patients treated as fourth-line died from HCC progression. According to modified RECIST, the objective response rate (ORR) and disease control rate (DCR) were $27.2 \%$ and $81.8 \%$, respectively. ORR and DCR by RECIST version 1.1 were $9.1 \%$ and $72.7 \%$, respectively. PFS at 3 months was $68.2 \%$. Drug discontinuation caused by adverse events (AEs) was reported in 7 patients treated as fourth-line and 1 patient as third-line. Pretreatment creatinine ( $\mathrm{Cr})$ and estimated glomerular filtration rate (eGFR) were significantly higher in patients with discontinuation owing to AEs $(P=0.01$ and $P=0.02)$. Urine protein-to-creatine ratio after 4 weeks was significantly higher in patients with impaired renal function than patients with preserved renal function $(0.93$ vs. $0.14 ; P=$ 0.017).

\section{Conclusion}

Ramucirumab therapy was found to have similar radiological responses between u-HCC patients with single and multiple experiences of TKI therapies. The pretreatment factors would be associated with the incidence of AEs during the therapy.

\section{Introduction}

Hepatocellular carcinoma (HCC) is one of the major causes of cancer-related deaths [1], and systemic therapies for unresectable HCC have been rapidly progressing. In several countries, various tyrosine kinase inhibitors (TKIs) such as sorafenib [2,3], lenvatinib [4], regorafenib [5], and cabozantinib [6] and the vascular endothelial growth factor receptor 2 (VEGFR-2) inhibitor ramucirumab $[7,8]$ have already been approved. Recently, the phase III IMbrave150 trial [9] has found that combined immunotherapy and VEGF inhibition with atezolizumab and bevacizumab had better clinical outcome and will become the new standard first-line therapy for advanced HCC. In this situation, it is necessary to reveal the clinical outcome of sequential therapies in real-world practice which was performed in different situations from the previous clinical trials. 
The REACH-2 trial [8] confirmed the efficacy of ramucirumab as second-line and all of the patients in this study had treatment with sorafenib. In Japan ramucirumab has been approved since June 2019. As a first-line agent, sorafenib and lenvatinib are equally recommended in many guidelines such as the Japan Society of Hepatology (JSH) [10], the American Association for the Study of Liver Disease [11], and the European Association for the Study of the Liver (EASL) [12]. Therefore, ramucirumab has administered in patients who were treated with lenvatinib. Moreover, as third-line or fourth-line, ramucirumab is administered to Japanese patients with unresectable HCC in real-world practice.

This study aimed to investigate the efficacy and safety of ramucirumab in real-world practice and analyze the clinical factors associated with adverse events (AEs) during the therapy.

\section{Methods}

\section{Patients}

A total of 20 patients with unresectable HCC received ramucirumab therapy at Musashino Red Cross Hospital from July 2019 to April 2020. The candidates for ramucirumab were patients with unresectable HCC, ECOG-PS 0-1, experiences with systemic chemotherapy for HCC, and alpha-fetoprotein (AFP) levels of $\geq 400 \mathrm{ng} / \mathrm{mL}$. Ramucirumab therapy was administered after discussions by the experts at a tumor board in the institution. Among them, 4 patients discontinued ramucirumab therapy within 2 weeks because of worsening general conditions caused by HCC progression. Finally, 16 patients were enrolled in this study. HCC diagnosis and evaluation of disease progression were based on guidelines proposed by JSH, ASSLD, or EASL. Written informed consent was obtained from all patients, and the ethics committee at the Musashino Red Cross Hospital approved the study in accordance with the Declaration of Helsinki.

\section{Treatment protocol}

Patients received intravenous ramucirumab ( $8 \mathrm{mg} / \mathrm{kg}$ ) for 1 hour every 14 days until disease progression or unacceptable toxicity. Dose reduction and interruption were done according to the guidelines provided by the manufacturer. Dynamic computed tomography was performed at baseline, at 4-8 weeks after ramucirumab administration, and every 6-10 weeks thereafter. Treatment response was reported based on response evaluation criteria in solid tumors (RECIST) and modified RECIST [13]. AFP level was also evaluated at baseline and every 4 weeks thereafter. AEs were evaluated according to the Common Terminology Criteria for Adverse Events (CTCAE) version 5.0 [14]. Drug discontinuation because of AEs included drug interruption of more than 6 weeks. As for the assessment of changes in liver function, the albumin-bilirubin (ALBI) score $[15,16]$ was analyzed at baseline and at 2, 4, and 8 weeks.

Statistical analysis

Overall survival (OS) was measured from the date of ramucirumab administration to the date of death from any cause. Patients who were lost to follow-up were censored at the last visit or contact. Patients who remained alive were censored on April 30, 2020. PFS was measured from the date of ramucirumab 
administration to the date of radiological tumor progression or death from any cause. Data were expressed as the median value (range). Fisher's exact test, Mann-Whitney $U$ test, paired $t$-test, KaplanMeier method, and log-rank test were used for statistical analyses. $P<0.05$ was considered statistically significant. Statistical analyses were performed with EZR (Saitama Medical Centre, Jichi Medical University, Shimotsuke, Japan) [17], a graphical user interface for R (The R Foundation for Statistical Computing, Vienna, Austria).

\section{Results}

Baseline patient characteristics

Baseline characteristics of these patients are presented in Table 1. The median ALBI score was -2.01 (range, -2.99 to -1.60 ) and baseline modified ALBI grades were 1, 2a, and $2 \mathrm{~b}$ in 1 (6.2\%), 2 (12.5\%), and 13 patients $(81.3 \%)$, respectively. HCC progression at the beginning of ramucirumab therapy was classified as BCLC stage B and C in $8(50.0 \%)$ and 8 patients $(50.0 \%)$, respectively. The major portal invasion was present in 4 patients (25.0\%). Extrahepatic metastasis was present in 6 patients $(37.5 \%)$. The median AFP level was $2296.5 \mathrm{ng} / \mathrm{mL}$ (range, 447.2-34327.7 ng/mL). Second-line, third-line, and fourth-line treatments were administered in 4 (25.0\%), 2 (12.5\%), and 10 patients (62.5\%), respectively. Among 4 patients who received ramucirumab as second-line, 3 patients were treated with lenvatinib as first-line and 1 patient participated in the double-blind clinical trial of lenvatinib or lenvatinib + pembrolizumab (NCT03713593). Among 2 patients who received ramucirumab as third-line, 1 patient was treated with sorafenib-lenvatinib and 1 patient received lenvatinib-sorafenib. Among 10 patients who received ramucirumab as fourth-line, 5 patients were treated with sorafenib-regorafenib-lenvatinib, 3 patients were treated with lenvatinib-sorafenib-regorafenib, 1 patient received sorafenib-lenvatinibregorafenib, and 1 patient received axitinib + avelumab (NCT03289533), cabozantinib (NCT03586973), and lenvatinib.

Table 1. Baseline characteristics 


\begin{tabular}{|ll|}
\hline & $\mathrm{n}=16$ \\
\hline Age (years), median (range) & $70(53-88)$ \\
\hline Sex, male/female (\%) & $11(68.8) / 5(31.2)$ \\
\hline Body weight (kg), median (range) & $60.6(49.6-91.8)$ \\
\hline Etiology HBV/HCV/alcohol/others (\%) & $3(18.8) / 11(68.8) / 1(6.2) / 1(6.2)$ \\
\hline Pretreatment ALBI score, median (range) & $-2.01(-2.99$ to -1.60) \\
Pretreatment modified ALBI grade 1/2a/2b (\%) & $1(6.2) / 2(12.5) / 13(81.3)$ \\
\hline ECOG-PS, 0/1/2 (\%) & $7(43.8) / 9(56.2) / 0(0)$ \\
\hline BCLC stage, A/B/C (\%) & $0(0) / 8(50.0) / 8(50.0)$ \\
\hline Major portal invasion, yes/no (\%) & $4(25.0) / 12(75.0)$ \\
\hline Extrahepatic spread, yes/no (\%) & $6(37.5) / 10(62.5)$ \\
\hline Baseline AFP concentration (ng/mL), & $2296.5(447.2-34327.7)$ \\
median (range) & \\
\hline Clinical course, & $4(25.0) / 2(12.5) / 10(62.5)$ \\
\hline second-line/third line/fourth-line (\%) & \\
\hline
\end{tabular}

Therapeutic efficacy

At the end of the data cutoff (April 30, 2020), the median duration of follow-up was 3.7 months (range, 1.9-9.4 months). The median course of ramucirumab was 5 (range, 2-18). During the observation period, 12 patients discontinued ramucirumab therapy because of progressive disease (PD) $(n=4)$ or AEs $(\mathrm{n}=8)$, and 3 patients died from HCC progression. There was no treatment-related death in this study. The median OS and PFS were not reached, and PFS at 3 months was $68.2 \%$ (Figure 1). In Child-Pugh A patients without macrovascular invasion $(n=8), 1$ patient died from HCC progression with bone metastasis. Radiological evaluation after ramucirumab administration was performed in 11 patients. With regard to the best antitumor response according to modified RECIST, no patient had complete response (CR), 3 patients had partial response (PR), 6 patients had stable disease (SD), and 2 patients had PD. The objective response rate (ORR) was $27.2 \%$ and disease control rate (DCR) was $81.8 \%$. With regard to the best antitumor response according to RECIST, no patient had CR, 1 patient had PR, 7 patients had SD, and 3 patients had PD. ORR and DCR were $9.1 \%$ and $72.7 \%$, respectively. AFP level decreased in 4 of 13 patients within 8 weeks after ramucirumab administration. Among 4 patients with decreased AFP, 2 patients showed PR and the remaining 2 patients were diagnosed as having SD by modified RECIST criteria. According to RECIST criteria, 1 patient showed PR and the remaining 3 patients were diagnosed as having SD. Among 4 patients with decreased AFP, AFP level at 4 weeks was decreased 
in 2 patients and the other 2 patients showed higher AFP at 4 weeks than pretreatment and decreased at 8 weeks after ramucirumab administration.

Changes in liver function

The changes in the ALBI score within 8 weeks are presented in Figure 2. The median ALBI scores at baseline and at 2, 4, and 8 weeks were -2.02 (range, -2.99 to -1.60 ), -1.91 (range, -2.69 to -1.31 ), -2.04 (range, -2.91 to -1.26 ), and -1.93 (range, -2.73 to -1.43 ), respectively. There were no significant differences in median ALBI score between baseline and at 4 weeks $(P=0.29)$ and between at baseline and at 8 weeks $(P=0.43)$.

Adverse events during ramucirumab therapy

AEs observed during ramucirumab therapy are presented in Table 2. Any grades of total AEs are observed in all patients. No infusion reaction was reported in all patients. Percentages of patients manifesting CTCAE grade 3 or more AEs were $43.8 \%$ for ascites and edema and $6.2 \%$ for increased total bilirubin (T$\mathrm{Bil})$. During the observation period, 8 patients discontinued ramucirumab therapy owing to AEs, as follows: 3 patients with edema, 3 patients with ascites, 1 patient with increased T-Bil, and 1 patient with cerebral infarction. In terms of cerebral infarction, the patient was male at the age of 80 years, received ramucirumab as fourth-line, and showed mild gait disorder after 2 courses of ramucirumab. Magnetic resonance imaging revealed multiple cerebral infarcts. The size of infarcts was small, and antithrombotic therapy and rehabilitation improved the patient to his condition before disease onset.

Table 2. Adverse events 


\begin{tabular}{|c|c|c|}
\hline & $\begin{array}{l}\text { Any grade } \\
\mathrm{n}(\%)\end{array}$ & $\begin{array}{l}\text { Grade } \geq 3 \\
\mathrm{n}(\%)\end{array}$ \\
\hline Hypertension & $8(50.0)$ & $0(0)$ \\
\hline HFSR & $3(18.8)$ & $0(0)$ \\
\hline Diarrhea & $4(25.0)$ & $0(0)$ \\
\hline Ascites & $6(37.5)$ & $4(25.0)$ \\
\hline Edema & $11(68.8)$ & $4(25.0)$ \\
\hline Decreased appetite & $8(50.0)$ & $0(0)$ \\
\hline Nausea & $1(6.2)$ & $0(0)$ \\
\hline Fatigue & $9(56.2)$ & $0(0)$ \\
\hline Increased $\mathrm{NH}_{3}$ & $3(18.8)$ & $0(0)$ \\
\hline Increased AST & $1(6.2)$ & $0(0)$ \\
\hline Increased ALT & $1(6.2)$ & $0(0)$ \\
\hline Increased T-Bil & $1(6.2)$ & $1(6.2)$ \\
\hline Proteinuria & $6(37.5)$ & $0(0)$ \\
\hline Thromboembolism & $1(6.2)$ & $0(0)$ \\
\hline Infusion reaction & $0(0)$ & $0(0)$ \\
\hline
\end{tabular}

Factors associated with adverse events during ramucirumab therapy

To investigate the higher incidence of AEs in this study compared with the REACH-2 study, we analyzed the baseline characteristics between patients with and without discontinuation of ramucirumab owing to AEs. Pretreatment age, creatinine $(\mathrm{Cr})$, and estimated glomerular filtration rate (eGFR) were significant factors associated with discontinuation owing to AEs. Pretreatment albumin, ALBI score, AFP, desgamma-carboxy prothrombin (DCP), and extrahepatic metastasis were not significantly different (Table 3). Taking these results into account, we focused on pretreatment renal function. In the REACH-2 study, creatinine clearance of $\geq 60 \mathrm{~mL} / \mathrm{min}$ was one of the inclusion criteria. We used eGFR instead of creatinine clearance. In this study, 10 patients with eGFR at $60 \mathrm{~mL} / \mathrm{min} / 1.73 \mathrm{~m}^{2}$ did not experience ascites during ramucirumab therapy. In contrast, all of 6 patients with impaired renal function (eGFR $<60 \mathrm{~mL} / \mathrm{min} / 1.73$ $\left.m^{2}\right)$ discontinued ramucirumab because of AEs such as severe ascites $(n=3)$, severe edema $(n=1)$, moderate edema with general fatigue $(n=1)$, and cerebral infarction $(n=1)$. There were no significant differences in age, body weight, pretreatment ALBI score, and pretreatment AFP between patients with preserved and impaired renal function. Six patients with impaired renal function discontinued ramucirumab because of AEs, and 3 of 4 patients with radiological evaluation were diagnosed as having 
SD by RECIST criteria. Pretreatment urine protein-to-creatine ratios between patients with preserved and impaired renal function were not significantly different; however, urine protein-to-creatine ratios were significantly higher in patients with impaired renal function than patients with preserved renal function at 4 weeks after ramucirumab administration ( 0.93 vs. $0.14, P=0.017)$.

Table 3. The comparison of baseline characteristics between patients with and without the discontinuation owing to AEs

\begin{tabular}{|c|c|c|c|}
\hline & $\begin{array}{l}\text { Patients without } \\
\text { discontinuation owing to AEs ( } \\
=8 \text { ) }\end{array}$ & $\begin{array}{l}\text { Patients with discontinuation } \\
\text { owing to AEs }(n=8)\end{array}$ & $\begin{array}{l}P \\
\text { value }\end{array}$ \\
\hline Age (median) & $67(53-86)$ & $72(70-88)$ & 0.04 \\
\hline Gender (male, \%) & $5(62)$ & $6(75)$ & 1.00 \\
\hline BW (kg, median) & $58.0(51.9-91.8)$ & $60.0(49.6-73.7)$ & 1.00 \\
\hline Alb (g/dL, median) & $3.3(2.9-3.9)$ & $3.2(2.8-4.3)$ & 0.39 \\
\hline ALBI score (median) & $-2.03(-2.57$ to -1.65$)$ & $-2.02(-2.99$ to -1.60$)$ & 0.59 \\
\hline $\mathrm{Cr}(\mathrm{mg} / \mathrm{dL}$, median) & $0.65(0.44-0.88)$ & $1.12(0.65-1.77)$ & 0.01 \\
\hline $\begin{array}{l}\text { eGFR }(\mathrm{mL} / \mathrm{min} / 1.73 \\
\mathrm{m}^{2} \\
\text { median })\end{array}$ & $81.1(66.2-109.9)$ & $51.1(30.4-95.0)$ & 0.02 \\
\hline $\begin{array}{l}\text { Urine protein-to- } \\
\text { creatine ratios } \\
\text { (median) }\end{array}$ & $0.15(0.03-0.63)$ & $0.14(0.02-5.61)$ & 0.56 \\
\hline $\begin{array}{l}\text { Pretreatment AFP } \\
\text { (ng/mL, median) }\end{array}$ & $3349(502.9-13372.8)$ & $1221(447.2-34327.7)$ & 0.96 \\
\hline $\begin{array}{l}\text { Pretreatment DCP } \\
\text { (mAU/mL, median) }\end{array}$ & $7643(37.8-41717.3)$ & $2169(266-8035.6)$ & 0.57 \\
\hline $\begin{array}{l}\text { Extrahepatic } \\
\text { metastasis } \\
\text { (yes, \%) }\end{array}$ & $4(50)$ & $2(25)$ & 0.68 \\
\hline
\end{tabular}

Efficacy and safety of ramucirumab as third-line or fourth-line therapy

To reveal the efficacy and safety of ramucirumab as second-line after lenvatinib, third-line, and fourth-line, we compared radiological findings and the incidence of discontinuation caused by AEs with the treatment background (Table 4).

Table 4. Radiological findings and incidence of discontinuation owing to AEs 


\begin{tabular}{|llllll|}
\hline & $\begin{array}{l}\text { PR by } \\
\text { RECIST }\end{array}$ & $\begin{array}{l}\text { SD by } \\
\text { RECIST }\end{array}$ & $\begin{array}{l}\text { PD by } \\
\text { RECIST }\end{array}$ & $\begin{array}{l}\text { Number of patients } \\
\text { without radiological } \\
\text { evaluation }\end{array}$ & $\begin{array}{l}\text { Number of } \\
\text { discontinuation } \\
\text { owing to AEs }\end{array}$ \\
\hline $\begin{array}{l}\text { Second-line after } \\
\text { lenvatinib }(\mathrm{n}=4)\end{array}$ & 1 & 2 & 0 & 1 & 0 \\
\hline Third-line $(\mathrm{n}=2)$ & 0 & 1 & 0 & 1 & 1 \\
\hline $\begin{array}{l}\text { Fourth-line }(\mathrm{n}= \\
10)\end{array}$ & 0 & 4 & 3 & 3 & 7 \\
\hline
\end{tabular}

\section{Discussion}

To the best of our knowledge, this is the first report on ramucirumab in the real-world practice including clinical information associated with AEs. Recently, Kuzuya et al [18] reported efficacy and safety of ramucirumab in Japanese patients with unresectable HCC. In the study, 8 of 10 patients received ramucirumab as second-line after lenvatinib and 2 patients were treated with ramucirumab as fourth-line. DCR at 6 weeks was $80 \%$ and the incidence of grade 3 AEs was $10 \%$ in the cohort. In our study, 10 of 16 patients received ramucirumab as fourth-line and DCR according to RECIST criteria was $72.7 \%$ in 11 patients with radiological evaluation. The median OS and PFS were not reached, and PFS at 3 months was $68.2 \%$. According to the results of REACH-2 study, the OS and PFS in ramucirumab group $(n=197)$ were 8.5 months and 2.8 months, and DCR evaluated by RECIST criteria was $59.9 \%$. All of the patients included in the REACH-2 trial received ramucirumab as second-line after sorafenib. The result of our study would reveal the efficacy of ramucirumab not only as second-line, but third or fourth-line, even though the number of the patients was small.

We found that the incidence of AEs in our study was higher than the REACH-2 trial and the recent report from Japan. Pretreatment age, $\mathrm{Cr}$, and eGFR were significantly different between patients with and without ramucirumab discontinuation owing to AEs; however, pretreatment ALBI score, AFP, and extrahepatic metastasis were not significant factors. Therefore, we hypothesized that renal function was associated with AEs because with aging, glomerular filtration rate and renal blood flow had been progressively decreasing. Moreover, it has already been reported that vascular and glomerular injury occurred with antiangiogenesis drugs targeting VEGF [19]. The mechanism of renal toxicity in bevacizumab, which is an anti-VEGF-A antibody, was associated with thrombotic microangiopathy $[20,21]$. In our study, 6 patients with impaired renal function received ramucirumab as fourth-line and all of them stopped ramucirumab because of AEs. Both impaired renal function and past treatment experiences with other TKIs which also targeted VEGF would be associated with a higher rate of ramucirumab discontinuation. DCR among patients with discontinuation because of AEs was $80 \%$. Four patients with preserved renal function received ramucirumab as fourth-line, and there was no severe $A E$ associated with ascites and edema. The appropriate treatment modification including dose and treatment schedule would contribute to long SD in patients with impaired renal function. 
As second-line after lenvatinib, 4 patients received ramucirumab and there was no severe AE. The radiological evaluation was performed in 3 patients. Two patients showed PR and the remaining 1 patient was diagnosed as having SD according to modified RECIST criteria. There was no patient who discontinued ramucirumab because of AEs in patients treated as second-line.

The limitations of our study are small number of patients, a retrospective cohort nature in a single center, and short observation period. However, the results revealed real-world situation, and some useful information about AEs during ramucirumab therapy for unresectable HCC could be found. Further studies in a large number of patients are necessary to confirm our results. Recently, the combination therapy with atezolizumab and bevacizumab was found to have excellent clinical outcome in the phase III trial and will become a first-line therapy for advanced HCC. In such situations, sequential therapies using molecular targeted agents would be more complicated and new treatment strategies should be established based on the results from the clinical trials and real-world data.

\section{Conclusions}

Ramucirumab therapy was found to have similar radiological responses in u-HCC patients with single or multiple experiences of TKI therapies. The pretreatment factors including renal function would be associated with the incidence of AEs during the therapy.

\section{Declarations}

\section{Availability of data and materials}

Original data and material are available from the corresponding author upon request.

\section{Acknowledgement}

This study was supported by a grant-in-aid from the Ministry of Health, Labor and Welfare, Japan.

\section{Declaration of Interest}

KT, MK and NI received advisory board fees and honoraria for speaker's bureau from Eisai, Bayer and Eli lilly.

\section{Funding}

This study was supported by a grant-in-aid from the Ministry of Health, Labor and Welfare, Japan.

\section{Ethical approval}

All procedures performed in studies involving human participants were in accordance with the ethical standards of the institutional and/or national research committee and with the 1964 Helsinki declaration and its later amendments or comparable ethical standards. 


\section{Informed consent}

Informed consent was obtained from all individual participants included in the study.

\section{References}

1. Bray F, Ferlay J, Soerjomataram I, et al. Global cancer statistics 2018: GLOBOCAN estimates of incidence and mortality worldwide for 36 cancers in 185 countries. CA Cancer J Clin. 2018;68(6):394-424.

2. Llovet JM, Ricci S, Mazzaferro V, et al. Sorafenib in advanced hepatocellular carcinoma. Engl J Med. 2008;359(4):378-390.

3. Cheng AL, Kang YK, Chen Z, et al. Efficacy and safety of sorafenib in patients in the Asia-Pacific region with advanced hepatocellular carcinoma: a phase III randomised, double-blind, placebocontrolled trial. Lancet Oncol. 2009;10:25-34.

4. Kudo M, Finn RS, Qin S, et al. Lenvatinib versus sorafenib in first-line treatment of patients with unresectable hepatocellular carcinoma: a randomised phase 3 non-inferiority trial. Lancet. 2018;391:1163-1173.

5. Bruix J, Qin S, Merle P, et al. Regorafenib for patients with hepatocellular carcinoma who progressed on sorafenib treatment (RESORCE): a randomised, double-blind, placebo-controlled, phase 3 trial. Lancet.2017; 389:56-66.

6. Abou-Alfa GK, Meyer T, Cheng AL, et al. Cabozantinib in Patients with Advanced and Progressing Hepatocellular Carcinoma. N Engl J Med. 2018;379: 54-63.

7. Park JO, Ryoo BY, Yen CJ, et al. Second-line ramucirumab therapy for advanced hepatocellular carcinoma (REACH): An East Asian and non-East Asian subgroup analysis. Oncotarget. 2016;7:75482-75491.

8. Zhu AX, Kang YK, Yen CJ, et al. Ramucirumab in advanced hepatocellular carcinoma and elevated alpha-fetoprotein following sorafenib (REACH-2): A randomised, double-blind, placebocontrolled phase 3 trial. Lancet Oncol. 2019;20:282-296.

9. Finn RS, Ducreux M, Qin S, et al. IMbrave150: A randomized phase III study of $1 \mathrm{~L}$ atezolizumab plus bevacizumab vs sorafenib in locally advanced or metastatic hepatocellular carcinoma. J Clin Oncol. 2018;36(15 suppl):TPS4141-TPS4141.

10. Kokudo N, Takemura N, Hasegawa K, et al. Clinical practice guidelines for hepatocellular carcinoma: The Japan Society of Hepatology 2017 (4th JSH-HCC guidelines) 2019 update. Hepatol Res. 2019;49(10):1109-1113.

11. Marrero JA, Kulik LM, Sirlin C, et al. Diagnosis, staging and management of hepatocellular carcinoma: 2018 practice guidance by the American Association for the Study of Liver Diseases. Hepatology. 2018;68:723-750.

12. European Association for the Study of the Liver. EASL Clinical Practice Guidelines: management of hepatocellular carcinoma. J Hepatol. 2018;69:182-236. 
13. Lencioni R, Llovet JM. Modified RECIST (mRECIST) assessment for hepatocellular carcinoma. Semin Liver Dis. 2010;30:52-60.

14. Oken MM, Creech RH, Tormey DC, et al. Toxicity and response criteria of the Eastern Cooperative Oncology Group. Am J Clin Oncol. 1982;5(6):649-655.

15. Johnson PJ, Berhane S, Kagebayashi C, et al. Assessment of liver function in patients with hepatocellular carcinoma: a new evidence-based approach-the ALBI grade. J Clin Oncol. 2014;20;33(6):550-558.

16. Hiraoka A, Kumada T, Tsuji K, et al. Validation of Modified ALBI Grade for More Detailed Assessment of Hepatic Function in Hepatocellular Carcinoma Patients: A Multicenter Analysis. Liver Cancer. 2019;8(2):121-129.

17. Kanda Y. Investigation of the freely available easy-to-use software 'EZR' for medical statistics. Bone Marrow Transplant. 2013;48:452-445.

18. Kuzuya $T$, Ishigami M, Ito $T$, et al. Initial Experience of Ramucirumab Treatment After Lenvatinib Failure for Patients With Advanced Hepatocellular Carcinoma. Anticancer Res. 2020; 40(4):20892093.

19. Abbas A, Mirza MM, Ganti AK, et al. Renal Toxicities of Targeted Therapies. Target Oncol. 2015;10(4):487-499.

20. Usui J, Glezerman IG, Salvatore SP, et al. Clinicopathological spectrum of kidney diseases in cancer patients treated with vascular endothelial growth factor inhibitors: a report of 5 cases and review of literature. Hum Pathol. 2014;45(9):1918-1927.

21. Eremina V, Jefferson JA, Kowalewska J, et al. VEGF inhibition and renal thrombotic microangiopathy. N Engl J Med. 2008;358(11):1129-1136.

\section{Figures}
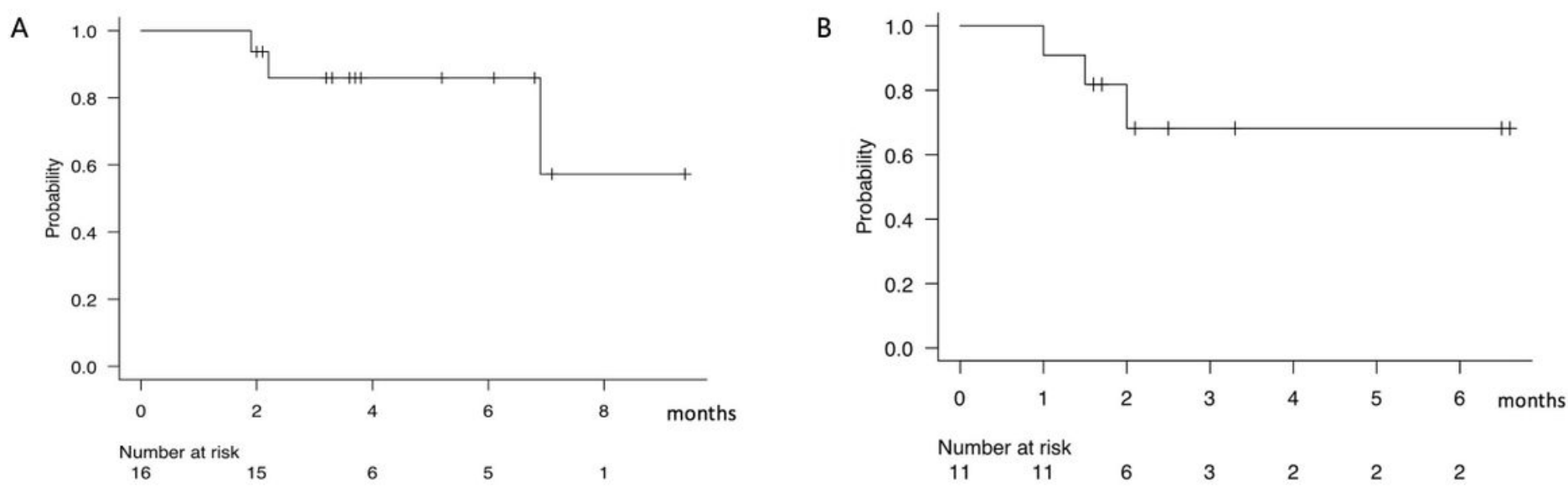

\section{Figure 1}


Overall survival (A) and progression-free survival (B) in all patients. A. Overall survival $(n=16)$ B. Progression-free survival $(n=11)$

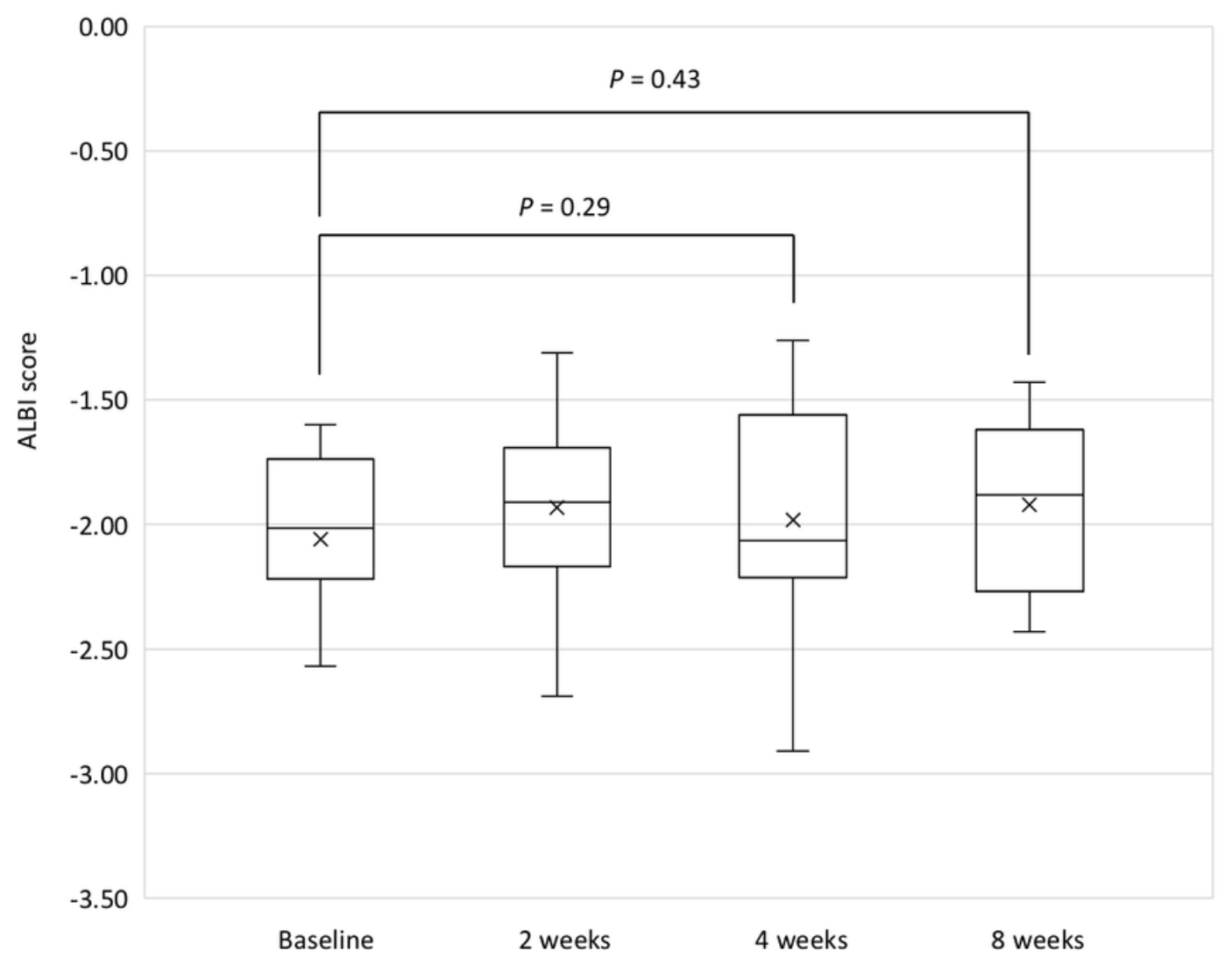

Figure 2

Changes in median ALBI score 